\title{
Circulating fibronectin to C-reactive protein ratio and mortality: a biomarker in COPD?
}

\author{
S.F.P. Man, L. Xing, J.E. Connett, N.R. Anthonisen, R.A. Wise, D.P. Tashkin, X. Zhang, \\ R. Vessey, T.G. Walker, B.R. Celli and D.D. Sin
}

ABSTRACT: The balance between inflammatory and repair processes is important in maintaining lung homeostasis in chronic obstructive pulmonary disease (COPD). The aim of the present study was to determine whether or not an integrated index of a biomarker involved in inflammation, C-reactive protein (CRP), and another involved in wound repair, fibronectin, may be a good measure to predict clinical outcomes in COPD.

Circulating blood levels of CRP and fibronectin were measured in 4,787 individuals with mild-tomoderate COPD who were prospectively followed for $>7$ yrs after blood collection as part of the Lung Health Study. To assess the balance between repair and inflammation, a simple ratio was calculated by dividing fibronectin levels by CRP levels and a Cox proportional hazards model was used to determine the relationship between this ratio and all-cause and disease-specific causes of mortality.

The relationship between the fibronectin to CRP ratio and all-cause mortality was L-shaped. There was an exponential decay in the adjusted hazard function (i.e. the risk of mortality) as the ratio decreased until a value of 148 was reached, beyond which point the hazard function did not change significantly. Similar results were observed for the risk of coronary and cardiovascular mortality.

Circulating fibronectin to CRP ratio is significantly associated with all-cause mortality of COPD patients. However, in contrast to other biomarkers, the relationship appears to be L-shaped (and not linear), suggesting a threshold at $\sim 150$. While promising, future studies are needed to validate this simple index as a biomarker in COPD.

KEYWORDS: Chronic obstructive pulmonary disease, C-reactive protein, fibronectin, inflammation, mortality, repair

hronic obstructive pulmonary disease (COPD) is a highly prevalent disease in the western world, affecting $10-15 \%$ of the adult population over the age of 45 yrs [1]. Within $15 \mathrm{yrs}$, COPD will be the third leading cause of mortality and the fifth leading cause of disability worldwide [2]. Unfortunately, there is a dearth of effective therapies that can prolong survival of COPD patients [3]. The development of novel therapeutic compounds for COPD has been impeded by a scarcity of robust intermediate end-points that can track disease progression and predict morbidity and mortality [4]. The pressing need for effective intermediate end-points for COPD therapeutic trials was highlighted by the American Thoracic Society (ATS)/European Respiratory Society (ERS) Task Force statement on outcomes for COPD pharmacological trials [5]. With the growing awareness of COPD as a systemic disease, there has been a shift in the emphasis of biomarker discovery towards blood specimens [6]. Serum or plasma biomarkers are attractive because blood, unlike bronchial washes or brushes, is readily available and the measurements can be easily standardised. To date, most of the attention has been focused on biomarkers associated with the systemic inflammatory pathway and some of these markers show promise, most notably C-reactive protein (CRP) [7-9] Another important but less studied pathway is wound repair [10]. Over- or underexpression of the reparative pathway can lead to disease states [11]. One possible blood biomarker of the reparative system is fibronection. Fibronectin is a high

For editorial comments see page 1426.

This article has supplementary material accessible from www.erj.ersjournals.com

AFFILIATIONS

For affiliations, please see the

Acknowledgements section.

CORRESPONDENCE

D.D. Sin, James Hogg iCAPTURE

Center, 1081 Burrard Street,

Vancouver, BC V6Z 1Y7, Canada.

Fax: 16048069274

E-mail: dsin@mrl.ubc.ca

Received:

November 142007

Accepted after revision:

August 272008

\section{SUPPORT STATEMENT}

This work was funded jointly by the Canadian Institutes of Health Research (CIHR; Ottawa, ON. Canada) and GlaxoSmithKline through a CIHR University-Industry Partnership program. The Lung Health Study was sponsored by a N01-HR-46002 contract from the Division of Lung Diseases of the National Heart, Lung, and Blood Institute (NHLBl; Bethesda, MD, USA). D.D. Sin holds the Canada Research Chair in Chronic Obstructive Pulmonary Disease (Ottawa) and is a senior scholar with the Michael Smith Foundation for Health Research (Vancouver, BC, Canada). J.E. Connett has received research support from the National Institutes of Health (Bethesda) for grants and contracts, specifically from the NHLBI and the National Institute for Allergy and Infectious Disease. J.E. Connett also serves on the data monitoring committees for seven studies sponsored by the NHLBI and the National Eye Institute.

\section{STATEMENT OF INTEREST}

A statement of interest for S.F.P. Man, R. Vessey, T.G. Walker, B.R. Celli and the study itself can be found at www.erj.ersjournals.com/ misc/statements.shtml 
molecular weight glycoprotein that is present in the body as two major isomers: a soluble form in the blood and an insoluble extracellular matrix isomer [12]. While blood fibronectin has many functions, its primary role is to promote wound repair following injury or infection by mediating cellular adhesion, motility, differentiation, apoptosis and haemostasis, and inducing the reticuloendothelial system [13]. This raises the possibility that an integrated measurable set of biomarkers that takes into account both CRP and fibronectin would perform better than CRP or fibronectin alone in COPD. In the present study, the potential usefulness of adding measurements of blood fibronectin to serum CRP data to create an integrated index of inflammation and repair to predict clinical outcomes in a cohort of patients with mild-to-moderate COPD was evaluated.

\section{METHODS}

\section{Study design}

The present study used data from 5,887 cigarette-smoking participants of the Lung Health Study (LHS). The participants were aged 35-60 yrs, had mild-to-moderate airflow obstruction on spirometry and were prospectively followed for $\leqslant 14.5 \mathrm{yrs}$ [14]. At entry, all participants demonstrated mild-to-moderate airflow obstruction as defined by a forced expiratory volume in one second (FEV1) of $<90 \%$ but $\geqslant 55 \%$ predicted, in the presence of a FEV1/forced vital capacity ratio of $<0.70$. Individuals who had a history of cancer (except carcinoma in situ or basal-cell carcinoma of the skin), myocardial infarction (in the previous $2 \mathrm{yrs}$ ), angina, heart failure, stroke (in the previous $2 \mathrm{yrs}$ ), renal failure, insulin-requiring diabetes mellitus, cirrhosis or other serious liver diseases, pulmonary embolism, disorders of the central nervous system, narrowangle glaucoma or any other major diseases that could have compromised follow-up were excluded from the cohort.

After enrolment and baseline measurements, the study participants were asked to visit the study centre annually for follow-up. At these visits, salivary cotinine levels were measured in order to verify smoking status of the participants objectively. Participants were categorised as sustained quitters if they were biochemically validated nonsmokers at each annual visit. Participants who were smokers at each annual visit were classified as continuous smokers. Those whose behaviour varied were classified as intermittent quitters.

The initial LHS involved 5 yrs of follow-up and the results of this phase of the study have been published previously [15, 16]. At the fifth annual visit, venipuncture was carried out on participants. There were 5,413 subjects who were alive and eligible for venipuncture at this visit. Of these, 4,803 provided serum samples (89\% of eligible participants), of which 4,787 had sufficient volume of serum for the fibronectin and CRP measurements. At this visit, the participants were also asked to consent for additional follow-up (LHS 3). During the follow-up of LHS 3, the participants' vital status was captured biannually. An independent mortality and morbidity committee (Minneapolis, MN, USA) reviewed death certificates, autopsy reports, relevant hospital records and summaries of interviews with attending physicians or eyewitnesses and assigned the causes of death for all participants who died during the study. These data were supplemented by linkage with a National Death Index (Hyattsville, MD, USA), which provided the date and cause of death for all study participants from the USA through until the end of 2001. Vital status was successfully determined for $98.3 \%$ of the participants [14]. Mortality endpoints were classified as follows: coronary heart disease (CHD); cardiovascular disease (CVD), which also included $\mathrm{CHD}$; lung cancer; other cancer; respiratory disease excluding lung cancer; other; and unknown. Since the blood samples were taken at year 5, individuals who died during the first 5 yrs of LHS follow-up and prior to blood sampling were excluded from the present analysis.

\section{Measurements of CRP and fibronectin}

After collection, the blood samples were separated into their various components, transferred to the LHS data-coordinating centre on dry ice and kept at $-70^{\circ} \mathrm{C}$ until use. The serum samples were thawed and CRP and fibronectin were determined using the SearchLight Proteome Array ${ }^{\mathrm{TM}}$ system (Pierce Biotechnology Inc., Rockford, IL, USA), a highly sensitive chemiluminescent multiplexed sandwich ELISA analyser that allowed simultaneous quantitative measurements of fibronectin and CRP from the same aliquot of serum.

\section{Statistical analysis}

To assess the joint effects of CRP and fibronectin, a simple ratio was calculated by dividing fibronectin levels by CRP levels in the systemic circulation, analogous to the use of the lowdensity lipoprotein cholesterol to high-density lipoprotein cholesterol ratio for assessing CVD risk in the general population [17]. The primary relationship of interest was between the serum fibronectin to CRP ratio and all-cause mortality. Other end-points were considered secondary in nature. All individuals who died or were lost to follow-up before visit 5 were excluded. Values of the fibronectin to CRP ratio were divided in quintiles from the lowest to the highest levels. The risk of all-cause mortality across the quintiles over the follow-up period was compared using a Cox proportional hazards model, in which the following covariates were adjusted for: age; sex; race; body mass index (BMI); pack-yrs of smoking; biochemically validated smoking status (i.e. continued smokers, sustained quitters or intermittent quitters); and percentage of predicted normal value FEV1 (in quintiles). To construct a parsimonious model, a stepwise selection method was used, in which variables were considered for further evaluation when their $p$-value was $\leqslant 0.25$ and retained in the model if their p-value was $\leqslant 0.15$ in the multivariate analysis. Similar methods were employed for the various causes of mortality. Logarithmic transformations were used for $\mathrm{CRP}$, fibronectin and the fibronectin to CRP ratio in order to achieve normality of distribution and to attenuate the influence of extreme outliers, which may artificially skew the data and produce misleading results. Continuous variables are presented as mean $\pm S D$ unless otherwise specified. A p-value $<0.05$ was considered to indicate statistical significance.

\section{RESULTS}

\section{Study participants}

The mean age of the study participants was $53.5 \pm 6.8$ yrs; 3,036 $(62.9 \%)$ were male and the average FEV1 at the fifth annual visit was $2.54 \pm 0.67 \mathrm{~L}(71.0 \pm 12.4 \%$ pred $)$. The average number of pack-yrs of smoking was $40.2 \pm 18.7 ; 1,363$ (28.0\%) were intermittent quitters and 2,607 (54.0\%) were continuous smokers at year 5 . The remainder were sustained quitters. 
The median (interquartile range (IQR)) fibronectin level was 316 (227-456) $\mathrm{mg} \cdot \mathrm{L}^{-1}$. The median (IQR) CRP level was $3.12(1.26-$ $7.30) \mathrm{mg} \cdot \mathrm{L}^{-1}$ and the median (IQR) fibronectin to CRP ratio was 107.2 (45.29-253.70). The characteristics of the study participants in quintiles of fibronectin to CRP ratio are shown in table 1. The fibronectin to CRP ratio was significantly related to the following variables: smoking status of the participants; age (for every 1-yr increment: $\beta$-coefficient $=-0.0075, p=0.019$ ); BMI (for every $1-\mathrm{kg} \cdot \mathrm{m}^{-2}$ increment: $\beta$-coefficient $=-0.0666, \mathrm{p}=0.0021$ ); pack-yrs of smoking (for every pack-yr: $\beta$-coefficient $=-0.0033$, $\mathrm{p}<0.0001$ ); systolic blood pressure (for every mmHg: $\beta$ coefficient $=-0.0036, \mathrm{p}=0.005$ ); and FEV1 (for every 1-L increment: $\beta$-coefficient $=0.4365, \mathrm{p}<0.0001$ ). Compared with sustained quitters, intermittent quitters and continued smokers had a decreased ratio (for intermittent quitters: $\beta$-coefficient $=-0.1255$, $p=0.021$; for continued smokers: $\beta$-coefficient $=-0.2729$, $\mathrm{p}<0.0001)$.

\section{Fibronectin to CRP ratio and mortality}

The median (range) duration of follow-up was 7.5 yrs (21 days to 7.5 yrs). During follow-up, there were 329 deaths, representing
$6.82 \%$ of the cohort. The level of serum fibronectin was negatively associated with all-cause mortality but this association failed to reach statistical significance (hazard ratio 0.84 for one-logarithmic increase in fibronectin level, 95\% confidence interval 0.70-1.00; $\mathrm{p}=0.055$; supplementary table 1$)$. Fibronectin levels were not associated with CHD- $(p=0.163)$, cancer- $(p=0.232)$ or respiratoryspecific mortality $(p=0.570)$. Conversely, serum CRP levels were positively associated with all-cause $(\mathrm{p}=0.004), \mathrm{CHD}-(\mathrm{p}=0.037)$ and CVD-specific mortality $(\mathrm{p}=0.017)$, as previously reported (supplementary table 2) [9]

The relationship between the ratio of fibronectin to CRP and all-cause mortality was L-shaped. The lowest risk of mortality was observed in the last three quintiles, while the highest risk was observed in quintile 1 (fig. 1 and table 2). This was further supported by an analysis in which the fibronectin to CRP ratio was assessed as a logarithmic continuous variable rather than in quintiles. The hazard function decreased as the ratio increased until a value of 148 was reached (fig. 1). Beyond this level, there was no significant change in the hazard function. The c-statistic (i.e. the area under the curve) was largest at 148, providing complementary evidence of a

TABLE 1 Clinical characteristics of study participants stratified in quintiles of fibronectin $(F)$ to $C$-reactive protein (CRP) ratio

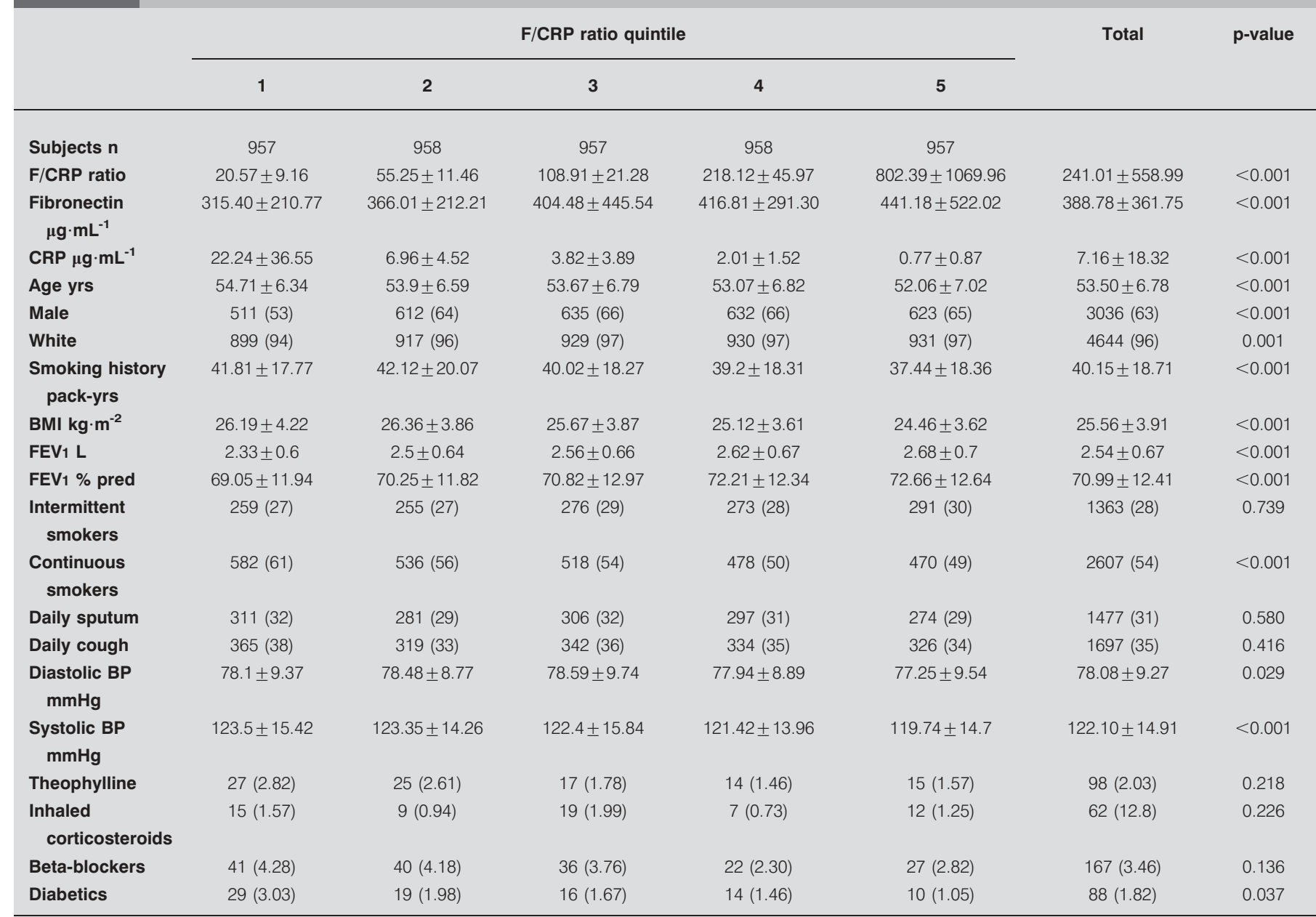

Data are presented as mean \pm SD or $n(\%)$, unless otherwise stated. BMI: body mass index; FEV1: forced expiratory volume in one second; \% pred: \% predicted; BP: blood pressure. 


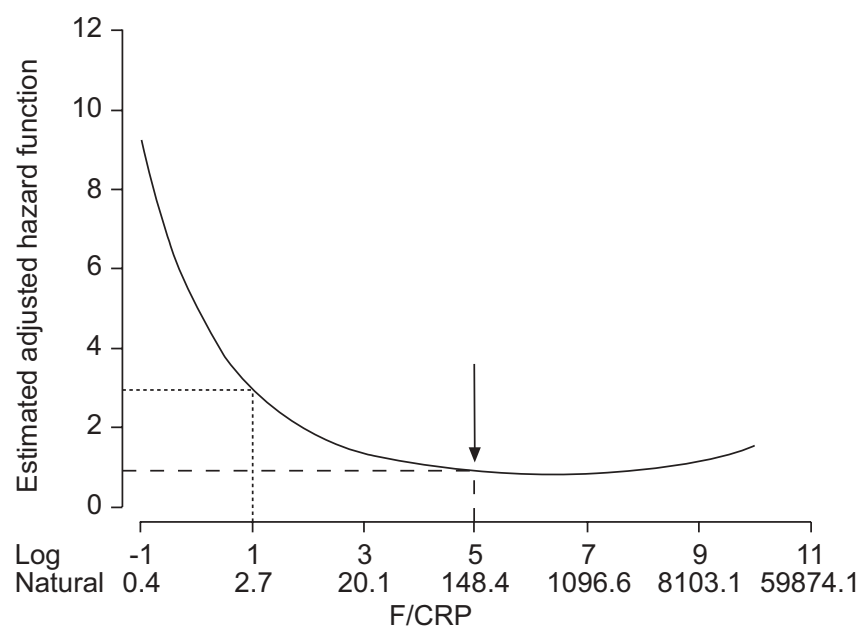

FIGURE 1. Fitted adjusted mortality curve evaluating the relationship between the fibronectin (F) to C-reactive protein (CRP) ratio and the hazard function for allcause mortality. The hazard function was generated using a Cox proportional hazards model in which the relationship between the F/CRP ratio (as a continuous variable) and all-cause mortality was evaluated, adjusted for various covariates. The fitted curve is presented, with both log and natural values of F/CRP. The arrow indicates a potential threshold at a natural F/CRP value of $\sim 150$, beyond which point the hazard function does not change significantly. Hazard ratios can be calculated from this graph by determining the hazard function at a particular value of F/CRP (e.g. 2.7; ‥) and dividing it by the hazard function of the reference F/CRP value (e.g. 148; ----). In this example, the hazard ratio would be 3 .

threshold at this point (supplementary fig. 2). Similarly, the risk of CHD and CVD mortality was highest in quintiles 1 and 2 (table 2 and fig. 2).

In table 3, the relationship between mortality and fibronectin to CRP ratio is summarised according to smoking categories at the time the serum samples were obtained. The relationship between fibronectin to CRP ratio and mortality held for intermittent and continued smokers but not for sustained quitters, although a lack of statistical power may have been responsible for this.

\section{Comparison of models}

The goodness of fit of the three models (CRP alone, fibronectin alone and fibronectin to CRP ratio models) was examined using the Baysian Information Criterion (BIC) and the Akaike Information Criterion (AIC; for further details see supplementary table 3 and the supplementary Methods). In general, models with the lowest AIC and BIC are preferred. For example, the AIC of the mortality model containing fibronectin alone was 5,268.32, while that for the model containing the fibronectin to CRP ratio was 5,254.87. Since the two models have the same number of parameters, the difference in AIC (13.45) is equivalent to a likelihood ratio of 832.98, indicating that the model containing the fibronectin to CRP ratio had better fit of data and greater discriminatory power than the model containing fibronectin alone $(\mathrm{p}<0.001$; see supplementary material for details). Indeed, for all end-points, the models that contained the fibronectin to CRP ratio in quintiles had the lowest AIC and BIC values, which suggests that the best model fit was achieved using fibronectin to CRP quintiles rather than fibronectin or CRP alone in quintiles.

\section{DISCUSSION}

In the present study, reduced levels of fibronectin relative to CRP were associated with increased all-cause and CVD mortality. Overall the relationship was L-shaped. There was an exponential decay of the hazard function as the ratio increased until a value of $\sim 150$ was reached. Beyond this point, the hazard function did not change significantly, suggesting a threshold at this value. Future studies will be needed to validate this threshold in a variety of different clinical settings (e.g. severe COPD populations). Similar L- or reverse L-shaped relationships have been described in other conditions. For instance, the risk of mortality is relatively constant until a BMI of $28 \mathrm{~kg} \cdot \mathrm{m}^{-2}$ is reached, beyond which point the risk increases sharply [18]. Similarly, the risk of CVD mortality increases exponentially only beyond a serum CRP level of $3 \mathrm{mg} \cdot \mathrm{L}^{-1}$ [19]. Although the current authors observed a relationship between blood levels of fibronectin/CRP and mortality, it is uncertain how pulmonary repair and inflammation are reflected in the circulating blood and how these

\begin{tabular}{|c|c|c|c|c|c|c|c|c|c|c|}
\hline \multirow{3}{*}{$\begin{array}{l}\text { Mortality } \\
\text { end-points }\end{array}$} & \multicolumn{10}{|c|}{ F/CRP ratio quintile } \\
\hline & \multicolumn{2}{|l|}{$1^{\#}$} & \multicolumn{2}{|l|}{$2^{\circ}$} & \multicolumn{2}{|l|}{$3^{\#}$} & \multicolumn{2}{|r|}{$4^{\circ}$} & \multicolumn{2}{|l|}{$5^{\#}$} \\
\hline & HR $(95 \% \mathrm{Cl})^{+}$ & Death rate & HR $(95 \% \mathrm{Cl})^{+}$ & Death rate & $\mathrm{HR}(95 \% \mathrm{Cl})^{+}$ & Death rate & $\mathbf{H R}^{+}$ & Death rate & $\mathrm{HR}(95 \% \mathrm{Cl})^{+}$ & Death rate \\
\hline All-cause & $2.08(1.46-2.98)$ & 103 (10.76) & $1.42(0.97-2.09)$ & $64(6.68)$ & 1.39 (0.95-2.05) & 64 (6.69) & 1 & $39(4.07)$ & $1.33(0.89-1.99)$ & $54(5.64)$ \\
\hline CHD & $5.07(1.46-17.61)$ & $17(1.78)$ & $5.17(1.50-17.76)$ & $16(1.67)$ & $1.62(0.39-6.77)$ & $5(0.52)$ & 1 & $2(0.21)$ & $3.80(1.05-13.83)$ & $10(1.04)$ \\
\hline CVD & $2.60(1.34-5.05)$ & 35 (3.66) & $2.42(1.24-4.72)$ & $31(3.24)$ & $1.01(0.46-2.22)$ & $13(1.36)$ & 1 & $10(1.04)$ & $1.78(0.86-3.69)$ & $18(1.88)$ \\
\hline Cancer & $1.46(0.98-2.17)$ & $66(6.90)$ & $0.98(0.63-1.52)$ & $41(4.28)$ & $1.21(0.80-1.84)$ & $51(5.33)$ & 1 & $37(3.86)$ & $0.97(0.61-1.53)$ & $36(3.76)$ \\
\hline Respiratory & $2.20(0.77-7.20)$ & $9(0.94)$ & $3.02(0.96-9.53)$ & $11(1.15)$ & $2.03(0.62-6.61)$ & $9(0.94)$ & 1 & $3(0.31)$ & $2.70(0.85-8.60)$ & $10(1.04)$ \\
\hline
\end{tabular}




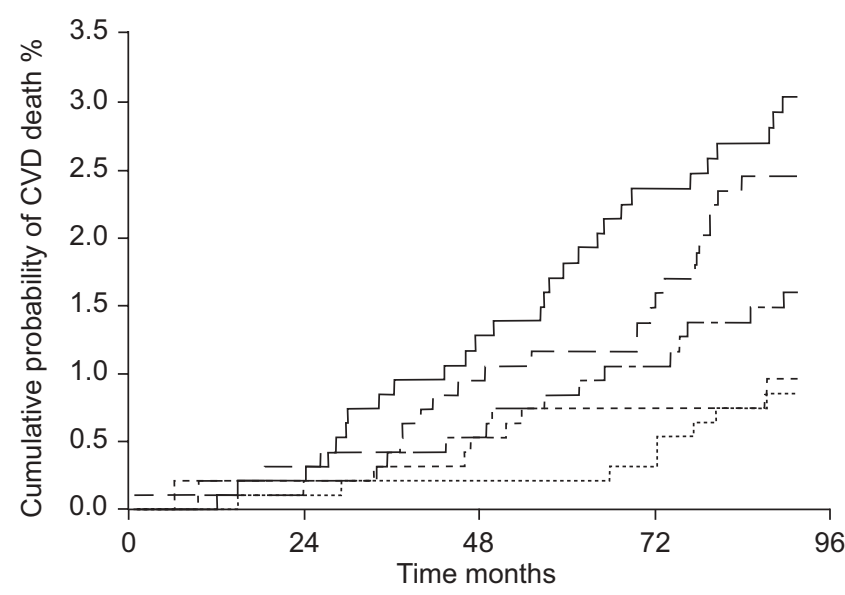

FIGURE 2. Cumulative cardiovascular mortality of participants with mild-tomoderate chronic obstructive pulmonary disease stratified according to quintiles of the fibronectin to C-reactive protein ratio. The survival curves are significantly different from each other ( $p<0.001$ by log-rank test). Quintiles 1, 3 and 5: $n=957$; quintiles 2 and $4: n=958$. CVD: cardiovascular disease. - - quintile $1 ;---$ quintile $2 ;-.-$ - quintile $3 ; \cdots$ : quintile $4 ;-\cdots-$ - quintile 5

molecules contribute to the increased mortality and morbidity of the subjects.

Both CRP and fibronectin play relevant roles in tissue injury and repair. CRP is an acute phase protein that is synthesised predominantly by hepatocytes. CRP activates complements as part of the innate immune response, enhances opsonisation of particles and microbes by macrophages and promotes endothelial activation [20]. Local overexpression of CRP amplifies tissue damage and increases infarction size in myocardium and brain [21]. Conversely, fibronectin is an adhesive glycoprotein that limits tissue injury and mediates cellular repair following tissue insult by promoting haemostasis and wound healing [22, 23]. Additionally, circulating fibronectin upregulates anti-apoptotic proteins such as B-cell leukaemia-2, thus preventing cellular death and limiting infarction size following ischemic injury [24]. Circulating fibronectin also enhances the ability of macrophages to phagocytose particles and microbes. Depletion of fibronectin increases lung deposits of blood-borne particles and microbes, which may cause disruptions of the lung microcirculation [25, 26]. Interestingly, CRP interacts with fibronectin by binding with it to initiate the repair process, which in turn creates a negative feedback loop [27].

To the current authors' knowledge, there are no published studies of the fibronectin/CRP ratio. However, there are examples of where biomarkers of repair (e.g. fibronectin) and inflammation (e.g. CRP or interleukin (IL)-6) have been associated with poor clinical outcomes. For example, reduced blood fibronectin is associated with coronary artery disease, which is generally associated with elevated CRP levels [28]. In meningococcal disease, children with the worst prognosis are those who have depressed blood fibronectin levels but elevated serum IL-6 levels [29]. Similarly, in sepsis syndrome, patients with the lowest blood fibronectin levels generally have the worst prognosis, while nearly all have elevated CRP levels [30, 31].

Although the local lung expression of fibronectin is usually increased [32], systemic (circulating) levels are, paradoxically, decreased in COPD [33]. The reduced levels are associated with increased symptoms and severity of disease [33]. The reduced systemic levels may reflect increased extravasation of circulating fibronectin to sites of local injury and accelerated turnover owing to chronic inflammation [34]. Cigarette smoking, which is the main cause of COPD, may further reduce fibronectin levels by inhibiting local production [35]. However, based on the present study, it is unclear whether the reduced fibronectin to CRP ratio was a cause or an effect of COPD.

There are several implications of the present study. First, it highlights the importance of considering both the inflammatory and repair pathways in assessing health risks in patients with COPD. CRP and fibronectin are linked with COPD progression but do not appear to be the main drivers of the progression. Secondly, unlike other markers of inflammation and repair, CRP and fibronectin are biomarkers uniquely useful for risk stratification of COPD patients because they are stable over long periods of time, have little diurnal variation and can be measured relatively inexpensively with commercially available assays [36, 37]. Traditionally, FEV1 has been used as the "gold standard" for determining prognosis in COPD patients. The present analysis shows that the fibronectin to CRP ratio provides incremental and independent prognostic

TABLE 3 Relationship between the fibronectin (F) to C-reactive protein (CRP) ratio and all-cause and specific causes of mortality in various smoking categories

\begin{tabular}{|c|c|c|c|c|}
\hline \multirow[t]{2}{*}{ Mortality end-points } & \multirow[t]{2}{*}{ All participants ${ }^{\#}$} & \multicolumn{3}{|c|}{ Smoking category } \\
\hline & & Sustained quitters & Intermittent quitters $^{+}$ & Continued smokers $^{\S}$ \\
\hline All-cause & $1.46(1.18-1.82)$ & $0.91(0.47-1.77)$ & $1.57(1.00-2.46)$ & $1.56(1.18-2.04)$ \\
\hline CHD & $2.81(1.56-5.05)$ & 4.29 (0.76-24.33) & $3.74(1.13-12.35)$ & $2.33(1.12-4.84)$ \\
\hline CVD & $2.26(1.53-3.33)$ & $2.17(0.53-8.81)$ & $2.36(1.06-5.23)$ & $2.25(1.40-3.60)$ \\
\hline Cancer & $1.17(0.91-1.52)$ & $0.92(0.42-2.01)$ & 0.97 (0.59-1.62) & $1.33(0.96-1.86)$ \\
\hline Respiratory & $1.30(0.71-2.37)$ & $2.16(0.35-13.33)$ & $2.61(0.78-8.72)$ & $0.85(0.38-1.87)$ \\
\hline
\end{tabular}

Data are presented as hazard ratio (95\% confidence interval), comparing F/CRP ratio quintiles 1 and 2 with quintiles 3 , 4 and 5 (reference), adjusted for age, sex and forced expiratory volume in one second (\% predicted). CHD: coronary heart disease; CVD: cardiovascular disease. ${ }^{\#}: n=4,809 ;{ }^{\bullet}: n=853 ;{ }^{+}: n=1,360 ;{ }^{\varsigma}: n=2,596$. 
information beyond that achieved by FEV1 alone, justifying the utility of this ratio in risk stratification and prognostication.

There were several limitations to the present study. First, serum CRP and fibronectin levels were measured at only one time-point. Therefore, the impact of changes in CRP and fibronectin levels over time on mortality of COPD patients is unknown. Any bias resulting from misclassification of the exposure variable would have diluted the relationship between the biomarkers and mortality. Thus, the present findings are probably conservative. Additionally, it is uncertain how the blood levels of these molecules are linked to the pathology of the lung disease in COPD. Notably, neither CRP nor fibronectin is lung specific; therefore, their blood levels may not correlate with what is happening in the lungs. Secondly, based on the current work, it is uncertain whether CRP or fibronectin is part of the causal pathway leading to mortality in COPD patients or just an epiphenomenon of other more salient molecules in the inflammatory and/or repair pathways. Thirdly, it is not clear whether the present data can be applied to other patient populations at risk for cardiac events (e.g. those with diabetes or obesity). Future studies will be needed in these specific populations in order to determine the potential utility of the fibronectin to CRP ratio in predicting cardiac events in these patients. Moreover, since only patients with COPD were studied in the present study, comparisons of the current findings with those in the general population are not possible. As recommended by the ATS/ERS Task Force, large population-based studies are needed in the future in order to establish "normative" values of blood biomarkers [5]. Finally, as the current study patients had mild-to-moderate COPD, the current findings may not be generalisable to patients with severe disease.

In conclusion, in a large cohort of well-characterised patients with mild-to-moderate chronic obstructive pulmonary disease, the fibronectin to $\mathrm{C}$-reactive protein ratio was found to be independently associated with mortality (especially cardiac mortality) after more than 7 yrs of follow-up, and the relationship was L-shaped, raising the possibility that this ratio may help clinicians and researchers with the risk stratification of patients with mild-to-moderate chronic obstructive pulmonary disease for prognosis and intervention.

\section{ACKNOWLEDGEMENTS}

The authors wish to thank the participants and investigators of the Lung Health Study and the three anonymous reviewers of the current manuscript, whose comments were invaluable in enhancing the quality and readability.

The authors' affiliations are as follows. S.F.P. Man and D.D. Sin: The James Hogg iCAPTURE Center for Cardiovascular and Pulmonary Research, St Paul's Hospital, and Pulmonary Division, Dept of Medicine, University of British Columbia, Vancouver, BC, Canada. L. Xing and X. Zhang: The James Hogg iCAPTURE Center for Cardiovascular and Pulmonary Research, St Paul's Hospital, Vancouver, BC, Canada. J.E. Connett: School of Public Health, University of Minnesota, Minneapolis, MN, USA. N.R. Anthonisen: Dept of Medicine, University of Manitoba, Winnipeg, MB, Canada. R.A. Wise: School of Medicine, Johns Hopkins University, Baltimore, MD, USA. D.P. Tashkin: School of Medicine,
University of California at Los Angeles, Los Angeles, CA, USA. R. Vessey and T.G. Walker: Discovery Medicine, High Throughput Biology and Biomedical Data Sciences, GlaxoSmithKline R\&D, King of Prussia, PA, USA. B.R. Celli: Pulmonary and Critical Care Division, Caritas St Elizabeth's Medical Center, School of Medicine, Tufts University, Boston, MA, USA.

\section{REFERENCES}

1 Halbert RJ, Natoli JL, Gano A, Badamgarav E, Buist AS, Mannino DM. Global burden of COPD: systematic review and meta-analysis. Eur Respir J 2006; 28: 523-532.

2 Murray CJ, Lopez AD. Alternative projections of mortality and disability by cause 1990-2020: Global Burden of Disease Study. Lancet 1997; 349: 1498-1504.

3 Sin DD, McAlister FA, Man SF, Anthonisen NR. Contemporary management of chronic obstructive pulmonary disease: scientific review. JAMA 2003; 290: 2301-2312.

4 Müller B, Tamm M. Biomarkers in acute exacerbation of chronic obstructive pulmonary disease: among the blind, the one-eyed is king. Am J Respir Crit Care Med 2006; 174: 848-849.

5 Cazzola M, MacNee W, Martinez FJ, et al. Outcomes for COPD pharmacological trials: from lung function to biomarkers. Eur Respir J 2008; 31: 416-469.

6 Malhotra S, Man SF, Sin DD. Emerging drugs for the treatment of chronic obstructive pulmonary disease. Expert Opin Emerg Drugs 2006; 11: 275-291.

7 Donaldson GC, Seemungal TA, Patel IS, et al. Airway and systemic inflammation and decline in lung function in patients with COPD. Chest 2005; 128: 1995-2004.

8 Hurst JR, Donaldson GC, Perera WR, et al. Use of plasma biomarkers at exacerbation of chronic obstructive pulmonary disease. Am J Respir Crit Care Med 2006; 174: 867-874.

9 Man SF, Connett JE, Anthonisen NR, Wise RA, Tashkin DP, Sin DD. C-reactive protein and mortality in mild to moderate chronic obstructive pulmonary disease. Thorax 2006; 61: 849-853.

10 Nathan C. Points of control in inflammation. Nature 2002; 420: 846-852.

11 Rennard SI. Inflammation and repair processes in chronic obstructive pulmonary disease. Am J Respir Crit Care Med 1999; 160: S12-S16.

12 Limper AH, Roman J. Fibronectin. A versatile matrix protein with roles in thoracic development, repair and infection. Chest 1992; 101: 1663-1673.

13 Mosesson MW, Amrani DL. The structure and biologic activities of plasma fibronectin. Blood 1980; 56: 145-158.

14 Anthonisen NR, Skeans MA, Wise RA, et al. The effects of a smoking cessation intervention on 14.5-year mortality: a randomized clinical trial. Ann Intern Med 2005; 142: 233-239.

15 Anthonisen NR, Connett JE, Enright PL, Manfreda J, Lung Health Study Research Group. Hospitalizations and mortality in the Lung Health Study. Am J Respir Crit Care Med 2002; 166: 333-339.

16 Anthonisen NR, Connett JE, Kiley JP, et al. Effects of smoking intervention and the use of an inhaled anticholinergic bronchodilator on the rate of decline of FEV1. The Lung Health Study. JAMA 1994; 272: 1497-1505. 
17 Manninen V, Tenkanen L, Koskinen P, et al. Joint effects of serum triglyceride and LDL cholesterol and HDL cholesterol concentrations on coronary heart disease risk in the Helsinki Heart Study. Implications for treatment. Circulation 1992; 85: 37-45.

18 Jee SH, Sull JW, Park J, et al. Body-mass index and mortality in Korean men and women. N Engl J Med 2006; 355: 779-787.

19 Ridker PM, Hennekens CH, Buring JE, Rifai N. C-reactive protein and other markers of inflammation in the prediction of cardiovascular disease in women. $N$ Engl J Med 2000; 342: 836-843.

20 Verma S, Yeh ET. C-reactive protein and atherothrombosis - beyond a biomarker: an actual partaker of lesion formation. Am J Physiol Regul Integr Comp Physiol 2003; 285: R1253-R1256.

21 Pepys MB, Hirschfield GM, Tennent GA, et al. Targeting Creactive protein for the treatment of cardiovascular disease. Nature 2006; 440: 1217-1221.

22 Kwon AH, Qiu Z, Hiraon Y. Effect of plasma fibronectin on the incisional wound healing in rats. Surgery 2007; 141: 254-261.

23 Matuskova J, Chauhan AK, Cambien B, et al. Decreased plasma fibronectin leads to delayed thrombus growth in injured arterioles. Arterioscler Thromb Vasc Biol 2006; 26 : 1391-1396.

24 Sakai T, Johnson KJ, Murozono M, et al. Plasma fibronectin supports neuronal survival and reduces brain injury following transient focal cerebral ischemia but is not essential for skin-wound healing and hemostasis. Nat Med 2001; 7: 324-330.

25 Baluna R, Sausville EA, Stone MJ, Stetler-Stevenson MA, Uhr JW, Vitetta ES. Decreases in levels of serum fibronectin predict the severity of vascular leak syndrome in patients treated with ricin A chain-containing immunotoxins. Clin Cancer Res 1996; 2: 1705-1712.

26 Cohler LF, Saba TM, Lewis E, Vincent PA, Charash WE. Plasma fibronectin therapy and lung protein clearance with bacteremia after surgery. I Appl Physiol 1987; 63 623-633.

27 Salonen EM, Vartio T, Hedman K, Vaheri A. Binding of fibronectin by the acute phase reactant $\mathrm{C}$-reactive protein. J Biol Chem 1984; 259: 1496-1501.

28 Zhang Y, Zhou X, Krepinsky JC, Wang C, Segbo J, Zheng F. Association study between fibronectin and coronary heart disease. Clin Chem Lab Med 2006; 44: 37-42.

29 Riordan FA, Bestwick K, Thomson AP, Sills JA, Hart CA. Plasma fibronectin levels in meningococcal disease. Eur J Pediatr 1997; 156: 451-453.

30 Herzum I, Renz H. Inflammatory markers in SIRS, sepsis and septic shock. Curr Med Chem 2008; 15: 581-587.

31 Grossman JE. Plasma fibronectin and fibronectin therapy in sepsis and critical illness. Rev Infect Dis 1987; 9: Suppl. 4, S420-S430.

32 Kranenburg AR, Willems-Widyastuti A, Moori WJ, et al. Enhanced bronchial expression of extracellular matrix proteins in chronic obstructive pulmonary disease. Am J Clin Pathol 2006; 126: 725-735.

33 Dong BR. [Clinical significance of fibronectin and protease inhibitors in chronic obstructive pulmonary diseases]. Zhonghua Jie He He Hu Xi Za Zhi 1989; 12: 343-346.

34 Ding YH, Lu YQ, Cheng JG. [Molecular degradation forms of plasma fibronectin in patients with chronic obstructive pulmonary disease]. Zhonghua Jie He He Hu Xi Za Zhi 1994; 17: 305-307.

35 Wang H, Liu X, Umino T, et al. Cigarette smoke inhibits human bronchial epithelial cell repair processes. Am J Respir Cell Mol Biol 2001; 25: 772-779.

36 Brubaker DB, Blick KE, Romine M. Four immunoassay methods and standards compared for measuring fibronectin. Clin Chem 1987; 33: 126-129.

37 Kimberly MM, Vesper HW, Caudill SP, et al. Standardization of immunoassays for measurement of high-sensitivity C-reactive protein. Phase I: evaluation of secondary reference materials. Clin Chem 2003; 49: 611-616. 